\title{
Stemness and inducing differentiation of small cell lung cancer $\mathrm{NCl}-\mathrm{H} 446$ cells
}

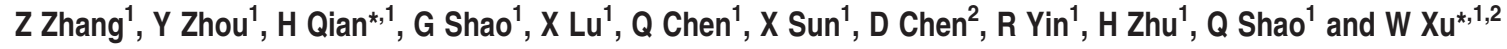

Small cell lung cancer (SCLC) accounts for nearly $15 \%$ of human lung cancers and is one of the most aggressive solid tumors. The SCLC cells are thought to derive from self-renewing pulmonary neuroendocrine cells by oncogenic transformation. However, whether the SCLC cells possess stemness and plasticity for differentiation as normal stem cells has not been well understood thus far. In this study, we investigated the expressions of multilineage stem cell markers in the cancer cells of SCLC cell line ( $\mathrm{NCl}-\mathrm{H} 446)$ and analyzed their clonogenicity, tumorigenicity, and plasticity for inducing differentiation. It has been found that most cancer cells of the cell line expressed multilineage stem cell markers under the routine culture conditions and generated single-cell clones in anchorage-dependent or -independent conditions. These cancer cells could form subcutaneous xenograft tumors and orthotopic lung xenograft tumors in BALB/C-nude mice. Most cells in xenograft tumors expressed stem cell markers and proliferation cell nuclear antigen Ki67, suggesting that these cancer cells remained stemness and highly proliferative ability in vivo. Intriguingly, the cancer cells could be induced to differentiate into neurons, adipocytes, and osteocytes, respectively, in vitro. During the processes of cellular phenotype-conversions, autophagy and apoptosis were two main metabolic events. There is cross-talking between autophagy and apoptosis in the differentiated cancer cells. In addition, the effects of the inhibitor and agonist for Sirtuin $1 / 2$ on the inducing osteogenic differentiation indicated that Sirtuin $1 / 2$ had an important role in this process. Taken together, these results indicate that most cancer cells of $\mathrm{NCl}-\mathrm{H} 446$ cell line possess stemness and plasticity for multilineage differentiation. These findings have potentially some translational applications in treatments of SCLC with inducing differentiation therapy.

Cell Death and Disease (2013) 4, e633; doi:10.1038/cddis.2013.152; published online 16 May 2013

Subject Category: Cancer

Lung cancer is now the leading cause of cancer death worldwide. Small cell lung cancer (SCLC) is a neuroendocrine subtype of lung cancer, accounting for approximately $15 \%$ of lung cancers, and is rarely curable. ${ }^{1,2}$ The cancer cells of SCLC may metastasize to lymph nodes or distant organs early due to the phenomenon of epithelial-mesenchymal transition (EMT). The chemotherapy and radiation therapy are the conventional treatments of SCLC. However, most patients treated with conventional therapies will relapse after a short period, resulting in a poor prognosis. ${ }^{3,4}$ For improving the survival of patients with SCLC, new therapies are urgently needed.

Differentiation therapy is a novel strategy for treating cancers, which is based on the concept that cancer cells arise from tissue stem cells and share the stemness and plasticity with normal stem cells. ${ }^{5-7}$ When the normal stem cells were arrested at an immature or less-differentiated state and suffered from oncogenic damages, they could transform into cancer cells. Differentiation therapy aims to induce the cancer cells to differentiate and maturate continuously by treatment with differentiation-inducing agents. ${ }^{8,9}$ Although most differentiation agents could not destroy the cancer cells directly, they could inhibit their proliferation and induce them to terminally differentiate and then undergo apoptosis. ${ }^{10-13}$ Differentiation therapy may drive a critical clinical and biological transition from a fatal cancer into one more amenable to treatment using conventional therapeutic approaches, which will improve the prognosis of patients with less-differentiated and highly aggressive tumor. ${ }^{14}$ Exploring the cellular origin, stemness, and plasticity of cancers in different organs will be helpful for inventing the inducing agents and methods to induce differentiation and apoptosis of the cancer cells. Understanding the differentiation mechanisms and the fates of cancer cells treated with inducing agents may eventually lead us to gain insights in curing cancers by differentiation therapy.

\footnotetext{
${ }^{1}$ School of Medical Science and Laboratory Medicine, Jiangsu University, Zhenjiang, Jiangsu, People's Republic of China and ${ }^{2}$ Affiliated Hospital, Jiangsu University, Zhenjiang, Jiangsu, People's Republic of China

*Corresponding author: H Qian or W Xu, School of Medical Science and Laboratory Medicine, Jiangsu University, 301 Xuefu Road, Zhenjiang, Jiangsu 212013, People's Republic of China. Tel: + 86511 85038215; Fax: + 86511 85038483; E-mail:Istmmmlst@163.com or icls@ujs.edu.cn

Keywords: small cell lung cancer; stemness; cancer stem cell; differentiation; autophagy; apoptosis

Abbreviations: SCLC, small cell lung cancer; EMT, epithelial-mesenchymal transition; ATRA, all-trans retinoic acid; TSA, trichostatin A; TUNEL, terminal deoxynucleotidyl transferase dUTP nick end labeling; ECM, extracellular matrix; NCAM, neural cell adhesion molecule; MMP-9, matrix metal proteinase 9; C/EBP $\beta$, CCAAT/enhancer-binding protein- $\beta$; PPAR $\gamma$, peroxisome proliferator-activated receptor gamma; FAS, fatty acid synthase; ATG, autophagy-related gene; HDAC, histone deacetylase; IBMX, 3-isobutyl-1-methyl-xanthine; NF-200, neuron marker neurofilament-200; H3K9, histone H3 lysine 9; FBS, fetal bovine serum; PVDF, polyvinylidene difluoride

Received 28.9.12; revised 27.3.13; accepted 3.4.13; Edited by Y Shi
} 
As cancer cells arise from stem cells, the degree of differentiation and malignancy of the cells mainly depend on the differentiating stage of origin stem cells at which the maturation arrest and oncogenic mutations occur. If maturation arrest of origin stem cells occurs early in the differentiating into multilineages, the cancer cells will be poorly differentiated and highly malignant. If the arrest occurs later in the differentiating into one cell lineage, the cancer cells will be well differentiated and less malignant. ${ }^{15}$

SCLC is a type of less-differentiated and highly aggressive tumor with a poor prognosis. SCLC cells were thought to derive from self-renewing pulmonary neuroendocrine progenitors, ${ }^{16,17}$ which display the transition from neuroendocrine into mesenchymal phenotype. ${ }^{18,19}$ However, whether the SCLC cells possess the stemness and plasticity as normal stem cells remains largely unknown. In this study, we have investigated the stemness, tumorigenicity, and plasticity for inducing differentiations of the SCLC NCl-H446 cells and explored the preliminary mechanisms of inducing differentiation and death of these cancer cells, such as regulatory effects of Sirtuin $1 / 2$ on osteogenic differentiation of the cancer cells and the cross-talking between autophagy and apoptosis during the differentiation processes.

\section{Results}

NCl-H446 cells expressed markers of various stem cells. The $\mathrm{NCl}-\mathrm{H} 446$ cells, cultured and passaged with DMEM medium containing $10 \%$ fetal bovine serum (FBS), could maintain stable morphological phenotype. When cultured on laminin-coated glass coverslips, the cancer cells attached and expanded in adherent monolayer on the substrate. However, as cultured at high cell density, the cancer cells could generate tumorspheres semiattached to culture plates. Immunofluorescence staining showed that most cancer cells of the cell line, whether grown in welladherent or in semi-adherent condition, could stably express cancer stem cell marker CD133, pluripotent stem cell markers Sall4 and Oct4, neural crest stem cell markers Nestin, neural cell adhesion molecule (NCAM), and $\mathrm{S} 100 \beta$, and mesenchymal stem cell (MSC) markers Vimentin, CD44, and CD105. The transcription factors Sall4 and Oct4 were mainly localized in the nuclei, meanwhile, the other marker proteins were located on the membrane and in the cytoplasm (Figure 1).

Most NCl-H446 cells could generate clones in anchorage-dependent or -independent conditions. The serial clonogenic assay showed that most original cancer cells of $\mathrm{NCl}-\mathrm{H} 446$ cell line cultured in the DMEM medium containing $10 \%$ FBS could generate primary single-cell clones (Figures $2 \mathrm{Aa})$, and the clone formation rate was $95 \%(54 / 57)$. The cancer cells derived from primary clones could generate single-cell subclones (Figures 2Ab), and the subclone formation rate was $100 \%(55 / 55)$. The cancer cells in these clones at different growth stages expressed neural crest marker NCAM (Figures 2Ac and d). Interestingly, once induced with all-trans retinoic acid (ATRA), the clones ceased expanding, and then the cells in these clones spread out and differentiated into neuron-like cells. The undifferentiated cells in the clones showed very weak immunofluorescence staining for neuron marker neurofilament-200 (NF200) (Figures 2Ae), however, the induced cells of the clones were strongly positive for NF-200 (Figures 2Af). When the primary $\mathrm{NCl}-\mathrm{H} 446$ cells were seeded in agarose, most cells could generate colonies in anchorage-independent conditions (Figures $2 \mathrm{Ba}$ and b). These colonies were positive for NCAM by immunofluorescence staining (Figures 2Bc and d). As isolated from agarose and cultured in adherent condition, the colonies attached fast, and then formed adherent monolayer cells, which expressed neural crest marker NCAM as well (Figures 2Be and f).

The $\mathrm{NCl}-\mathrm{H} 446$ cells possessed stable tumorigenicity. When $1 \times 10^{4}$ passaged cells derived from original $\mathrm{NCl}-\mathrm{H} 446$ cells were subcutaneously injected into the nude mice, subcutaneous xenograft tumors would generate (Figures 3ac). The cancer cells of subclones derived from subcutaneous xenograft tumors could secondly generate orthotopic xenograft tumors in the lungs (Figures $3 d-f$ ). The cancer cells seeding in subcutaneous tissues and lungs could form cancer nests, which grew as clones expanded in vitro. The nests grew bigger and bigger, and then generated solid tumors. Most cancer cells in the xenograft tumors expressed multilineage stem cell markers. The surface and cytoplasm proteins CD133, CD44, Nestin, Vimentin, and matrix metal proteinase 9 (MMP-9) were located on the cell membrane and in the cytoplasm (Figures $4 \mathrm{a}-\mathrm{e}$ ), meanwhile, the transcription factors Sox-2, Sall4, c-Myc, and the proliferation cell nuclear antigen Ki67 were mainly distributed in the nuclei of the cancer cells (Figures $4 \mathrm{f}-\mathrm{i}$ ).

The NCl-H446 cells could be induced by neurogenic differentiation, autophagy and apoptosis. When treated with trichostatin A (TSA), $\mathrm{NCl}-\mathrm{H} 446$ cells changed into neuron-like appearance with many neurites interconnected as a network (Figure 5a). The differentiated cancer cells were strongly positive for immunofluorescence staining of neural markers BM88 (Figure 5b) and NF-200 (Figure 5c). The positive cells of immunofluorescence staining for proliferation cell marker Ki67 was decreased gradually (Figure 5d). However, the positive cells of immunofluorescence staining for the autophagy marker Beclin-1 (Figure 5e) and the apoptotic cells for terminal deoxynucleotidyl transferase dUTP nick end labeling (TUNEL) staining (Figure 5f) were increased gradually. The changes of proliferation index and apoptotic index indicated that the cancer cells ceased proliferation and underwent apoptosis (Figure $5 \mathrm{~g}$ ). Western blotting showed that TSA-treated cancer cells overexpressed NF-200 and BM88(CEND1). The level of acetyl-histone H3 lysine $9(\mathrm{H} 3 \mathrm{~K} 9)$ in these cells was raised due to inhibition of histone deacetylases (HDACs) by TSA as well (Figure 5h). In addition, in these differentiated cells the increasing accumulations of autophagy-related proteins, Atg7 and Beclin-1, which were cleaved, and the conversion of LC3-I to LC3-II were also observed. Meanwhile, the levels of pro-apoptotic proteins, Bax, pro-caspase-3, and cleaved caspase-3, were raised; however, the levels of $\mathrm{Bcl}-2$ and $\mathrm{Ki} 67$ were decreased gradually (Figure $5 \mathrm{i}$ ). 

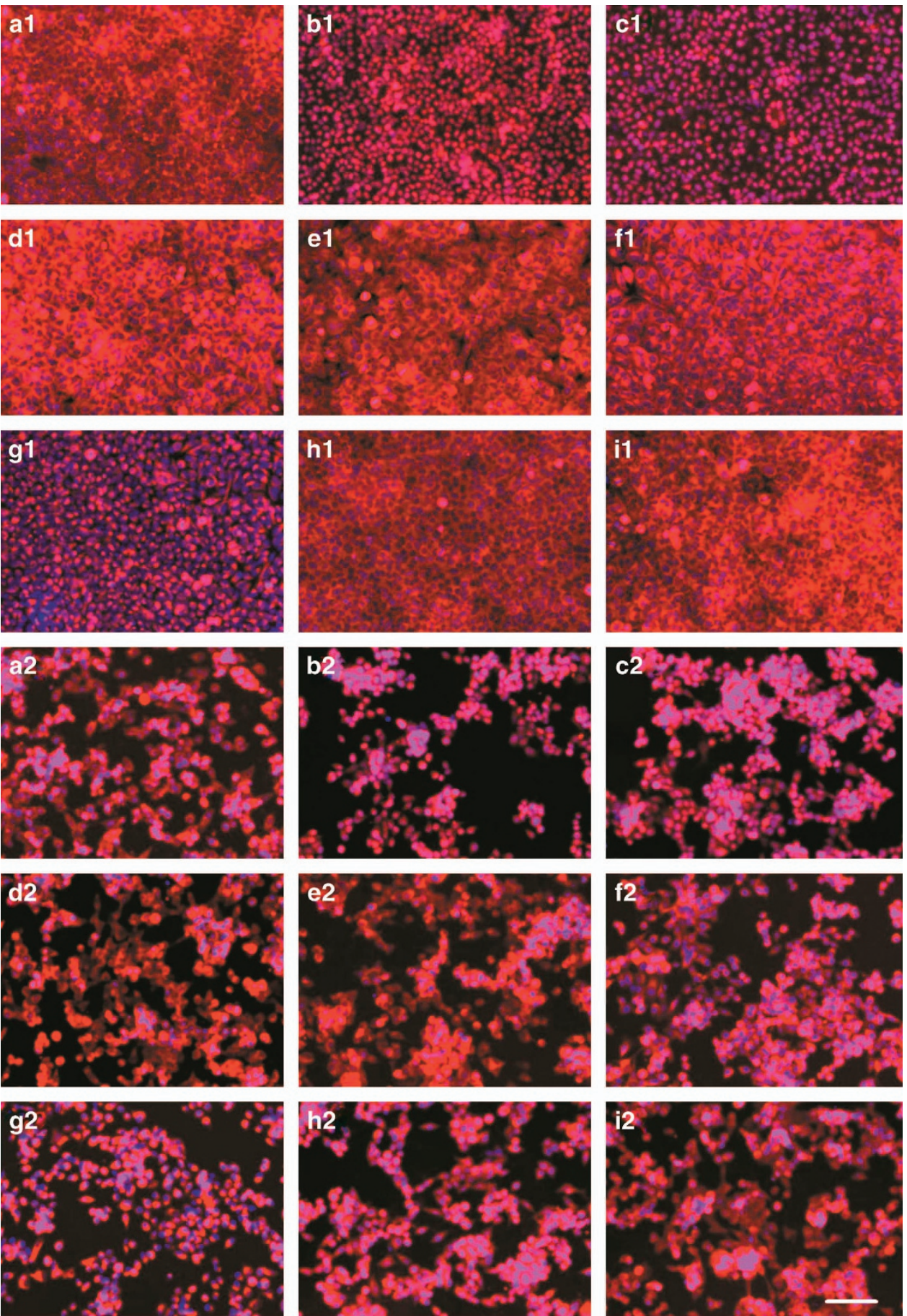

Figure 1 The $\mathrm{NCl}-\mathrm{H} 446$ cells expressed stem cell markers. When cultured on laminin-coated glass coverslips, the cancer cells could attach well and expanded in adherent monolayer on the substrate (a1-i1). However, as cultured at high cell density, the cancer cells could generate tumorspheres semiattached to culture plates (a2-i2). These cells could express stem cell markers: (a) CD133; (b) Sall4; (c) Oct4; (d) Nestin; (e) NCAM; (f) S100 $\beta$; (g) Vimentin; (h) CD44; and (i) CD105. Immunofluorescence staining with Cy3, counterstaining nuclei with Hoechst 33342. Scale bar, $50 \mu \mathrm{m}$

The $\mathrm{NCl}-\mathrm{H} 446$ cells could be induced by adipogenic differentiation. To evaluate the adipogenic ability of $\mathrm{NCl}$ H446 cells, the cancer cells were cultured in adipogenic induction medium/maintenance medium for three cycles. The Oil Red O staining showed that these differentiating cancer cells produced and accumulated extensive lipid droplets in the cytoplasm, leading to collapse of the cancer cells finally. Western blotting showed that after inducing differentiation, the cells overexpressed adipogenic regulatory proteins, including CCAAT/enhancer-binding protein- $\beta$ (C/EBP $\beta)$, peroxisome proliferator-activated receptor gamma (PPAR $\gamma$ ), fatty acid synthase (FAS), and adiponectin (Figure 6). 


\section{A}
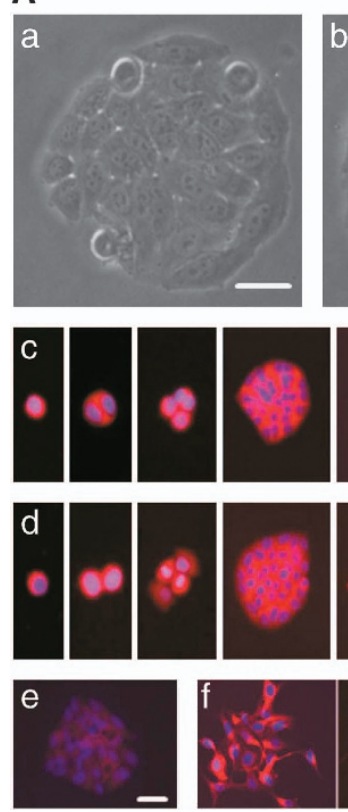
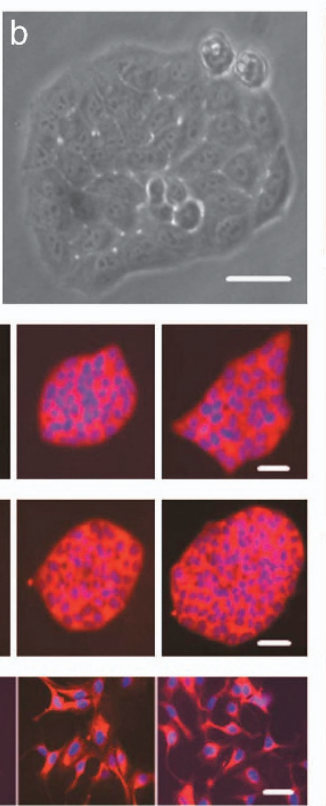

B
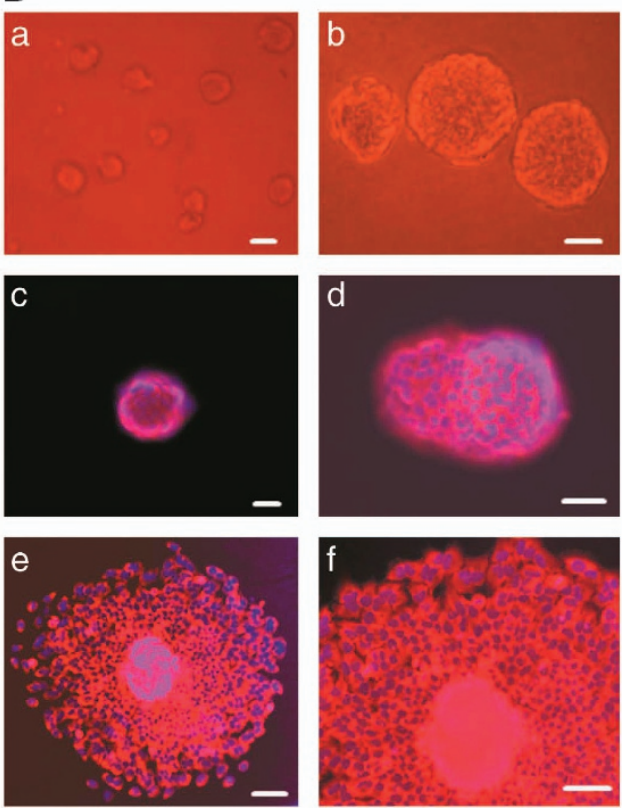

Figure 2 The generation of clones derived from NCl-H446 cells in anchorage-dependent or -independent conditions. (A) The generation of clones in anchorage-dependent condition. The outline of the clones was sharp; the cancer cells in these clones grew in confluent condition: (a) primary clone and (b) subclone. The cells of these clones at different growth stages expressed NCAM: (c) primary clones and (d) subclones. The undifferentiated subclone cells showed very weak immunofluorescence signal for neuron marker NF200 (e). When treated with ATRA (for 2, 3, and 5 days, respectively), the subclones ceased expansion, and the cells in these clones spread out and differentiated to neuron-like cells with very strong immunofluorescence staining for NF-200 (f). (B) The generation of colonies in anchorage-independent condition. Most cells seeded in agarose could generate colonies in anchorage-independent conditions: (a) the colonies grew for 2 weeks and (b) the colonies grew for 3 weeks. The cancer cells in these colonies were positive for NCAM by immunofluorescence staining: (c) one colony grew for 2 weeks and (d) two colonies grew for 3 weeks at different depth, which were fusing. As isolated from agarose and cultured in adherent condition, the spherical colony attached and expanded fast to form adherent monolayer cells, which expressed NCAM as well: (e) an intact colony and expanded cells from it and (f) magnified image of e. Immunofluorescence staining with Cy3, counterstaining nuclei with Hoechst 33342. Scale bar, $20 \mu \mathrm{m}$ (a and b); $50 \mu \mathrm{m}$ (c-f)
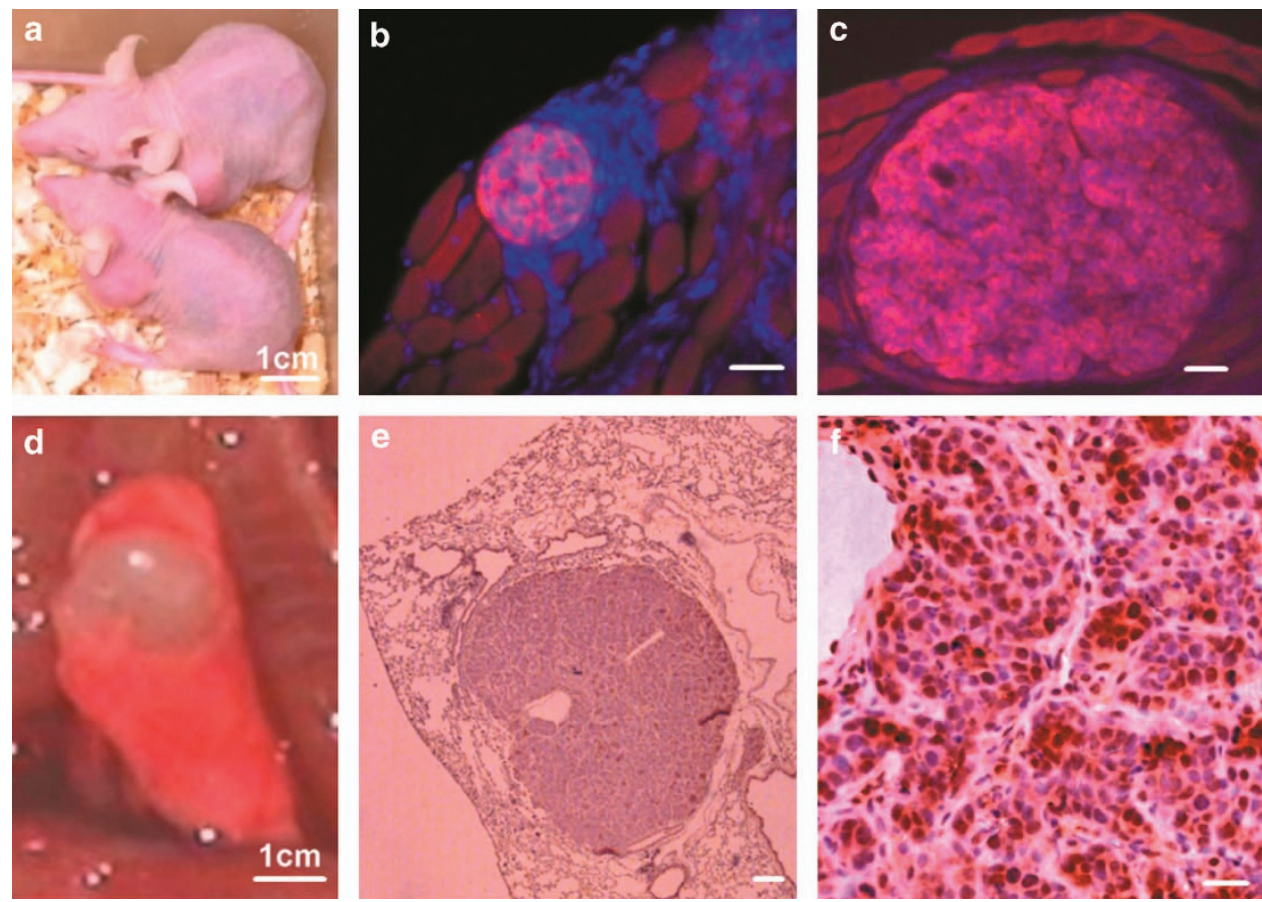

Figure 3 The NCl-H446 cells could form subcutaneous and orthotopic xenograft tumors. (a) The mice bearing subcutaneous xenograft tumors. (b and $\mathbf{c}$ ) The subcutaneous xenograft tumors, at different growth stages, were positive for NCAM. (d) The lung implanted with orthotopic xenograft tumor. (e) The intact orthotopic lung xenograft tumor grew as sphere. (f) Most cancer in the orthotopic lung xenograft tumor were positive for Ki67. Immunofluorescence staining with Cy3, counterstaining nuclei with Hoechst 33342 in $\mathbf{b}$ and $\mathbf{c}$. Immunohistochemistry with DAB visualizing Ki67-positive cells, counterstaining nuclei with hematoxylin in e and f. Scale bar, $1 \mathrm{~cm}(\mathbf{a}$ and $\mathbf{d}) ; 50 \mu \mathrm{m}$ (others) 

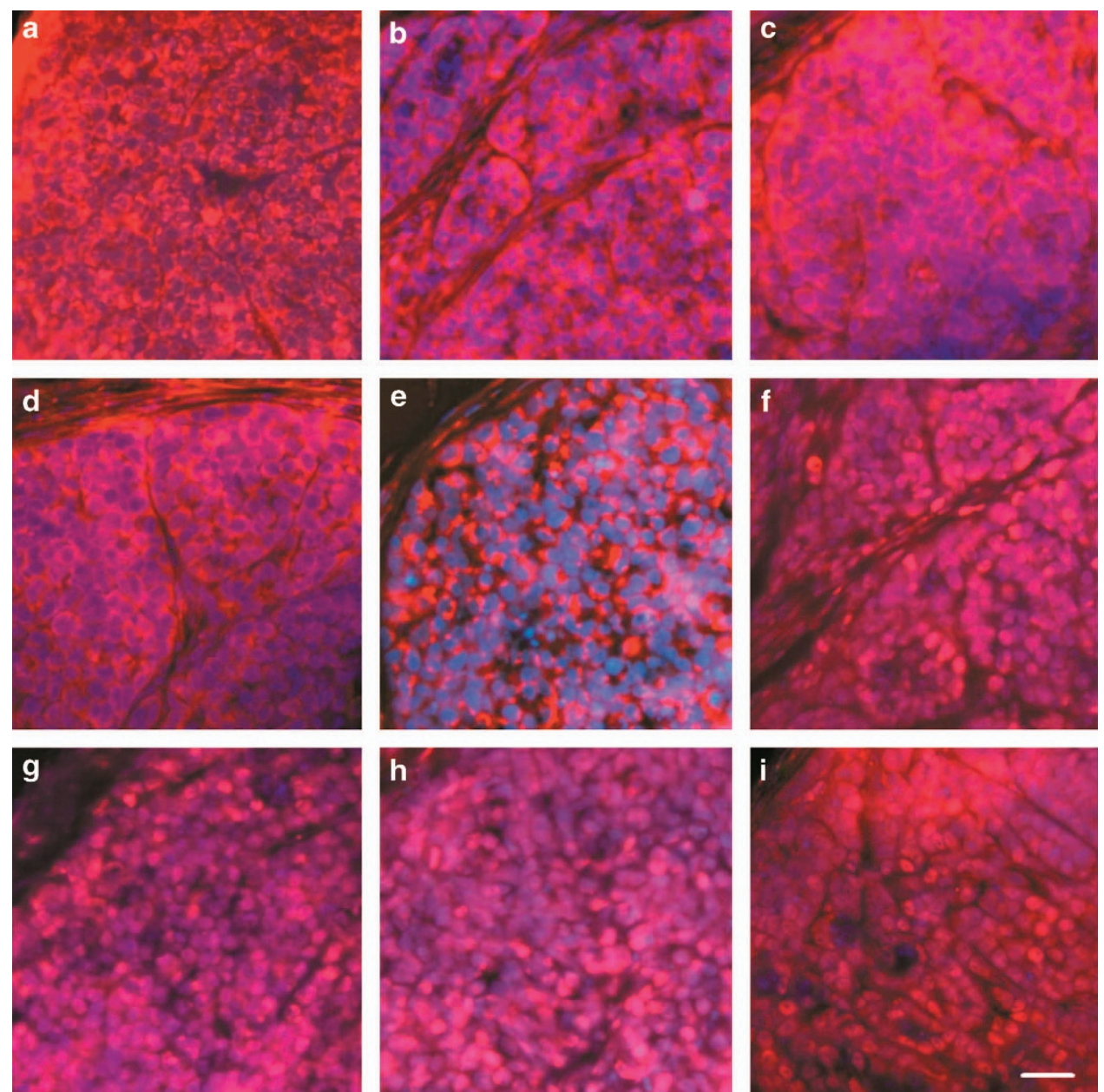

Figure 4 The cancer cells in xenograft tumor expressed multilineage stem cell markers. These stem cell markers included cancer stem cell markers CD133 (a) and CD44 (b), neural stem cell marker Nestin (c), MSC marker Vimentin (d), metastasis-associated protein MMP-9 (e), stem cell transcription factors Sox-2 (f), Sall4 (g), and c-Myc (h), and proliferation cell nuclear antigen Ki67 (i). Immunofluorescence staining with Cy3, counterstaining nuclei with Hoechst 33342 . Scale bar, $50 \mu \mathrm{m}$

The $\mathrm{NCl}-\mathrm{H} 446$ cells could be induced by osteogenic differentiation. To examine whether $\mathrm{NCl}-\mathrm{H} 446$ cells could differentiate into osteoblasts, the cancer cells were cultured in osteogenic induction medium for 3 weeks. After 1 week of induction, the cancer cells changed into big multiform osteoblast-like cells. The osteoblast-like cancer cells showed strong activity of alkaline phosphatase (Figure 7a). The Alizarin Red S staining demonstrated that calcium deposition and mineralization on the surface of the differentiating cancer cells increased gradually (Figure 7b). The inhibitor of Sirt1/2, cambinol could inhibit osteogenic differentiation, however, the agonist for Sirt1, resveratrol, could promote this differentiation (Figure 7c). Western blotting showed that after inducing differentiation, the cancer cells overexpressed the autophagy-related proteins in parallel to expressions of the apoptosis markers, meanwhile, the bone matrix proteins, including collagen-I and osteocalcin, were increased gradually (Figure 7d). During the differentiation process, the osteogenic regulatory proteins inducing Runx2 and Foxo3a were upregulated, whereas the adipogenic regulatory protein PPAR $\gamma$ was downregulated. Cambinol could inhibit expressions of the osteogenic regulatory proteins, and resveratrol could promote expressions of these proteins; The expression of Sirt1 was changed gently; however, the activity of Sirt1/2 showed changing obviously by detection of the actylated tubulin- $\alpha$ (Figure 7e).

Xenograft tumors could be induced to calcify and ceased growth by osteogenic differentiation therapy. After the complete DMEM medium (as control) or osteogenic induction medium was orthotopically injected into subcutaneous xenograft tumors and surrounding tissues, respectively, for 4 weeks, the molybdenum-target X-ray examination showed that the xenograft tumors in the control animals were expanded extensively, and the density of the tumor masses was uniform (Figure 8a). However, in the osteogenic differentiation group, the tumor masses were smaller than those in the control group, the density of the masses was not uniform, in the center of the masses, and high-density components were detected, demonstrating calcification. In the periphery of the masses, low-density foci were showed, suggesting the necrosis in the tumors 
(Figure $8 b$ ). Staining of the tissue sections of the xenograft tumors with Alizarin Red S showed that calcium deposition and mineralization in the tumors of induced animals were increased gradually (Figures $8 \mathrm{c}$ and d). More importantly, treatment with inducing osteogenic differentiation could inhibit growth of the tumors (Figures $8 \mathrm{e}$ and $\mathrm{f}$ ).
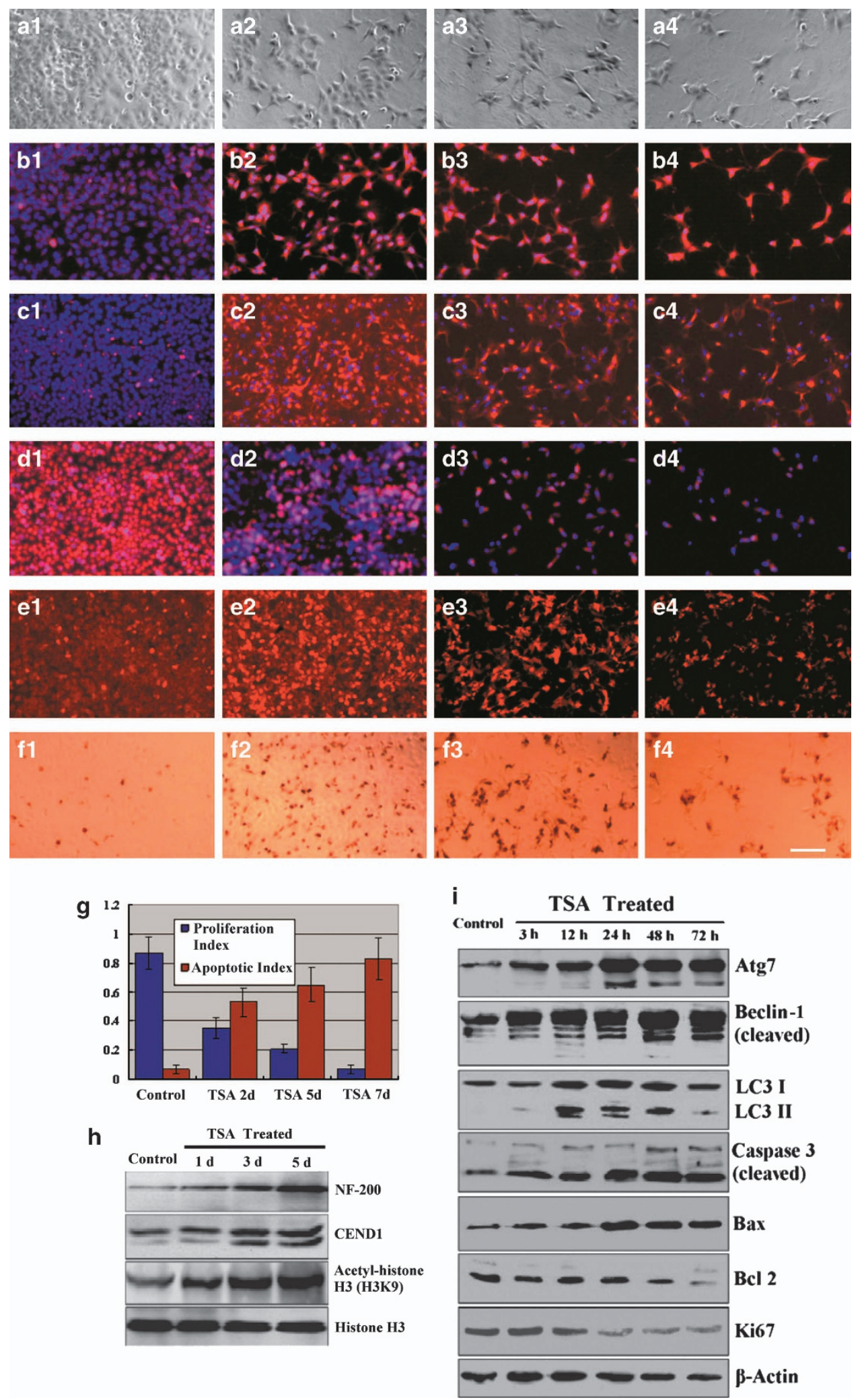


\section{Discussion}

SCLC is a neuroendocrine subtype of lung cancer possessing highly aggressive and metastatic ability. Understanding the biological mechanisms of these malignant clinical behaviors will contribute to improving clinical therapy for curing SCLC. In this study, the stemness, malignancy, and inducing differentiation of SCLC cancer cells were studied using $\mathrm{NCl}$ H446 cell line as a cellular model. As solid tumors contain cancer cells and normal tissue cells, such as tumorassociated fibroblasts and MSCs, it is difficult to separate and purify cancer cells from solid tumors for researching the cellular biocharacteristics. In contrast, cancer cell lines do not contain any normal stem cells, so that they could be attractive
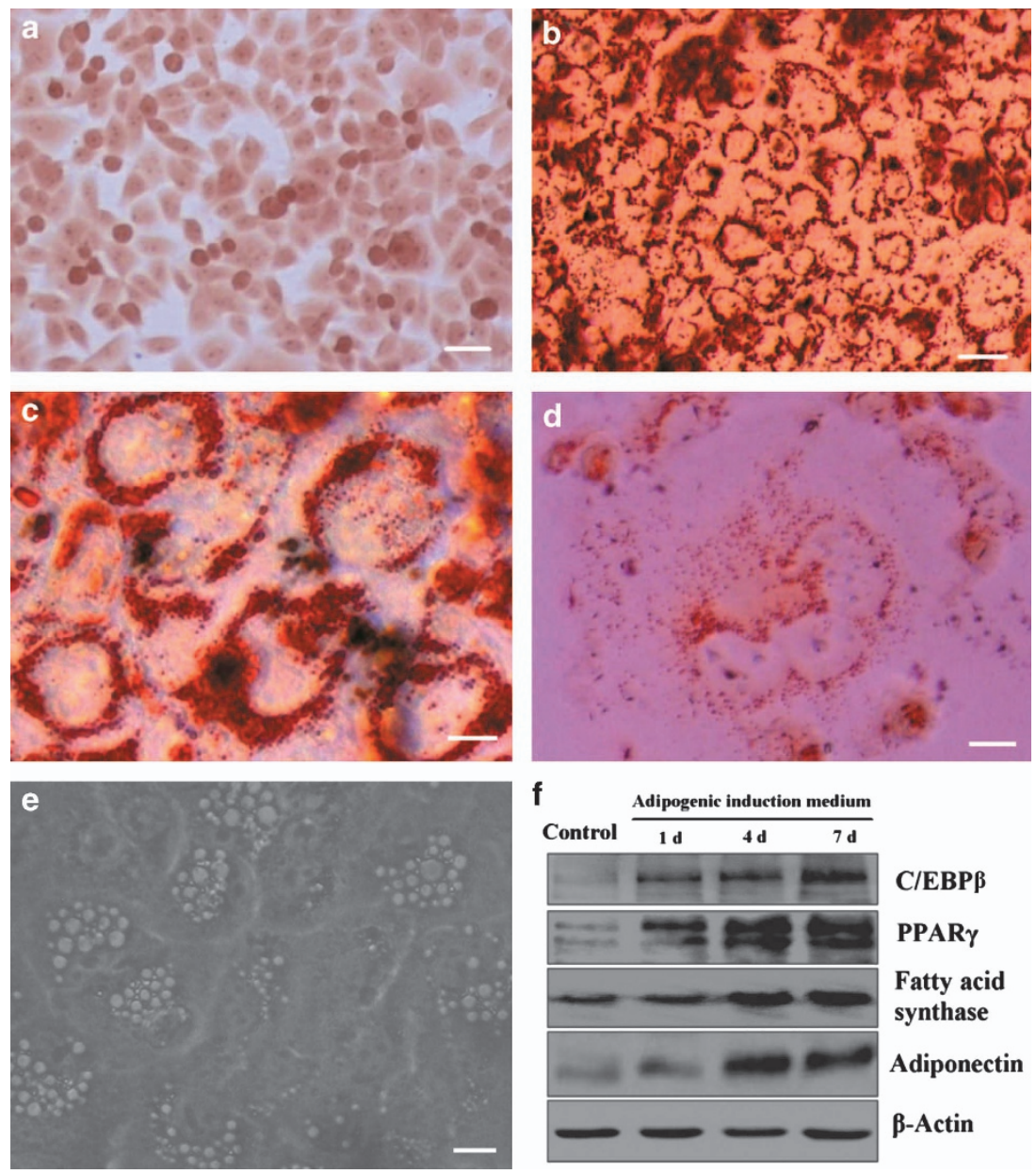

Figure 6 Induced adipogenic differentiation of $\mathrm{NCl}-\mathrm{H} 446$ cells. The Oil Red O staining showed that when cultured in adipogenic induction/maintenance medium for two cycles, the cancer cells could produce a lot of lipid droplets: (a) the control group and (b) showing lipid droplets in induced cancer cells. (c) The magnified image of the cells in $\mathbf{b}$, showing these lipid droplets more obviously. (d) Inducing for three cycles, these cancer cells collapsed due to accumulation of too much lipid droplets in the cytoplasm. (e) The phase contrast images showed bright lipid droplets accumulated in the mature adipocyte-like cancer cells, inducing for two cycles. Western blotting showed that after inducing differentiation, the cells overexpressed C/EBP $\beta$, PPAR $\gamma$, FAS, and adiponectin (Figure $5 \mathrm{e}$ ). Scale bar, $20 \mu \mathrm{m}$ (a and $\mathbf{b}) ; 5 \mu \mathrm{m}$ (c-e)

Figure 5 Induced neurogenic differentiation of $\mathrm{NCl}-\mathrm{H} 446$ cells. After treatment with $\mathrm{TSA}, \mathrm{NCl}-\mathrm{H} 446$ cells changed into neuron-like appearance with many neurites interconnected as a network (a1, control; a2-a4, TSA treated for 2, 5, and 7 days, respectively). The differentiated cancer cells were strongly positive for immunofluorescence staining of neuron markers BM88 (b1, control; b2-b4, TSA treated for 2, 5, and 7 days, respectively) and NF-200 (c1, control; c2-c4, TSA treated for 2, 5, and 7 days, respectively). Treatment with TSA could inhibit proliferating of the cancer cells, so that the number of these cells was decreased gradually due to death of differentiated cells (d1: in control group, most nuclei of cancer cells were positive for Ki67; d2-d4: after treatment with TSA for 2, 5, and 7 days, respectively, fewer and fewer nuclei of the cells were positive). After treatment with TSA, the autophagic cells stained for the autophagy marker Beclin-1 were increased gradually (e1, control; e2-e4, TSA treated for 2, 5, and 7 days, respectively). TUNEL staining showed that treatment with TSA could induce the cancer cells to undergo apoptosis (f1: in control group, fewer nuclei of cancer cells were positive for TUNEL staining; f2-f4: after treatment with TSA for 2, 5, and 7 days, respectively, more and more nuclei of the cells were positive, meanwhile, the number of total cells was decreased gradually due to apoptosis). (g) The proliferation index and apoptotic index indicated that the cancer cells ceased proliferation and underwent apoptosis. (h) Western blotting showed that TSA-treated cancer cells overexpressed NF-200 and BM88 (CEND1). The level of acetyl-H3K9 in these cells was raised due to inhibition of HDACs by TSA as well. In addition, in these differentiated cells the increasing accumulations of autophagy-related proteins, Atg7 and Beclin-1, which were cleaved, and the conversion of LC3-I to LC3-II were also detected; meanwhile, the levels of pro-apoptotic proteins, Bax, pro-caspase-3, and cleaved caspase-3, were raised. (i) However, the levels of Bcl-2 and Ki67 were decreased gradually. (a1-a4) The phase contrast images. (b1-e4) Immunofluorescence staining with Cy3, counterstaining nuclei with Hoechst 33342. (f1-f4) Visualizing the apotoptic nuclei with DAB by TUNEL staining. Scale bar, $100 \mu \mathrm{m}$ 

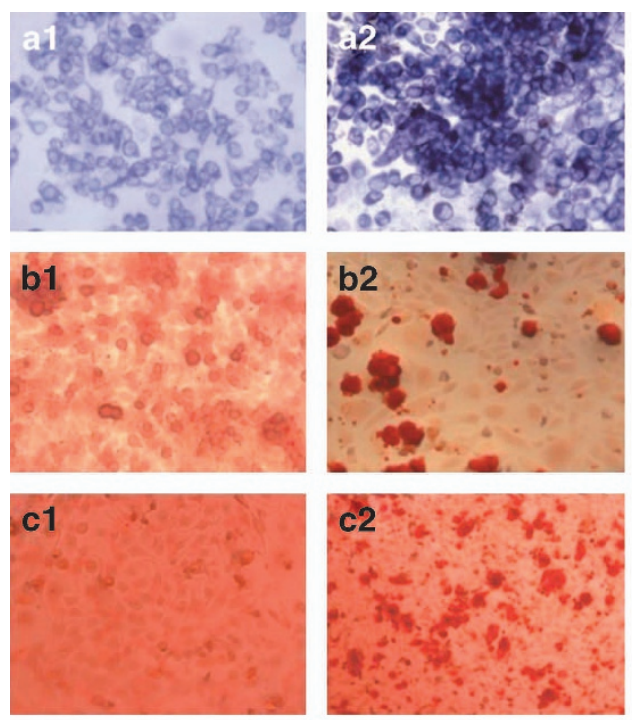

d

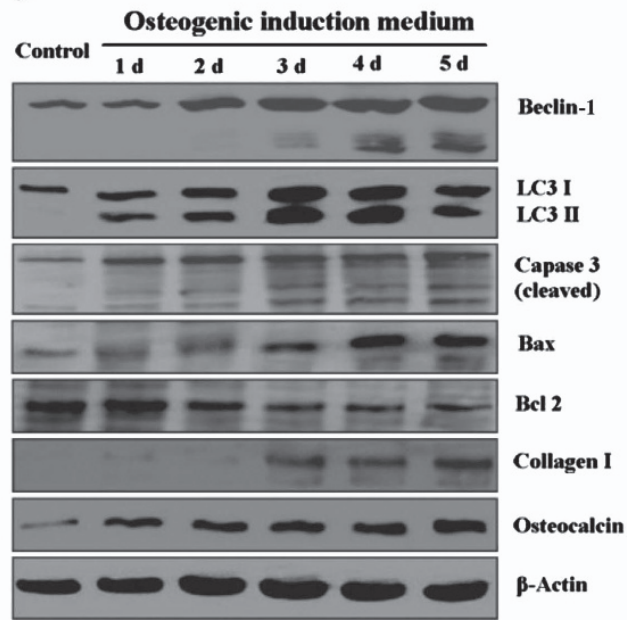

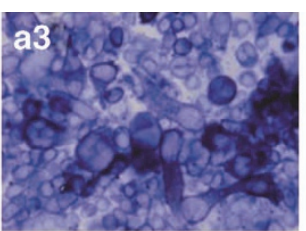
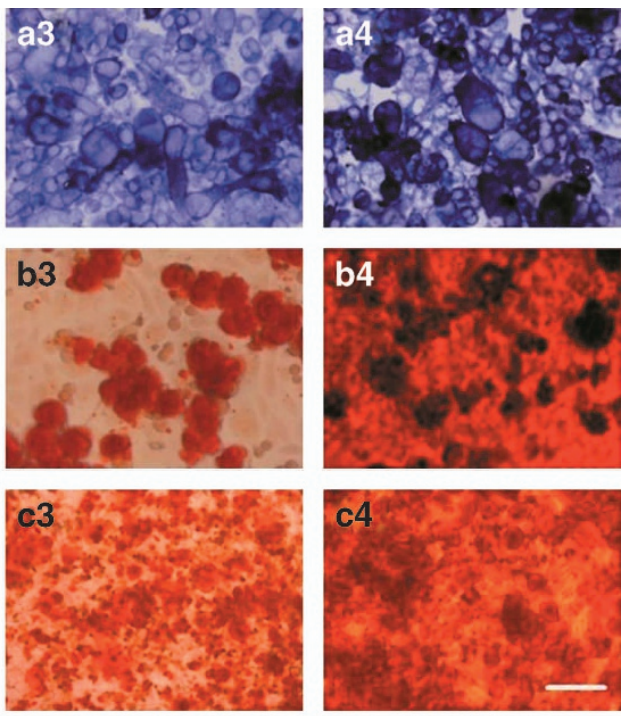

e

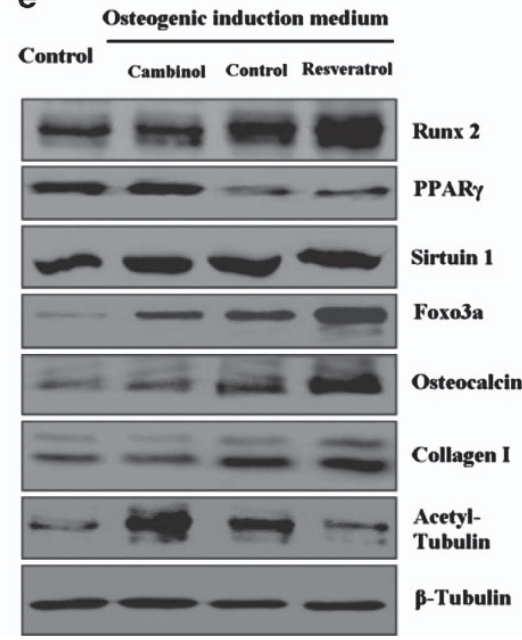

Figure 7 Induced osteogenic differentiation of $\mathrm{NCl}-\mathrm{H} 446$ cells. After cultured in osteogenic induction medium, the cancer cells changed into bigger multiform osteoblastlike cells. The osteoblast-like cancer cells showed strong activity of alkaline phosphatase (a1, control group; a2, inducing for 3 days; a3, inducing for 1 week; and a4, inducing for 2 weeks). The Alizarin Red S staining showed the mineralized bone nodules on the surface of the osteocyte-like cancer cells (b1, control group; b2, inducing for 1 week; b3, inducing for 2 weeks; and b4, inducing for 3 weeks). The inhibitor of Sirt1/2, cambinol, could inhibit osteogenic differentiation, however, the agonist for Sirt1, resveratrol, could promote osteogenic differentiation (c1, cultured in DMEM medium containing $10 \%$ FBS for 1 week; $\mathbf{c 2}$, cultured in osteogenic indution medium containing $100 \mu \mathrm{M}$ cambinol for 2 weeks; $\mathbf{c 3}$, cultured in osteogenic indution medium for 2 weeks; and c4, cultured in osteogenic indution medium containing $100 \mu \mathrm{M}$ resveratrol for 2 weeks). Western blotting showed that after inducing differentiation, the levels of autophagy-related proteins (Atg7 and Beclin), were increased, and these proteins were cleaved dynamicly. LC3-I was processed into LC3-II as the indicator of autophagy, in parallel to changing levels of the apoptosis markers (caspase-3, Bax, and Bcl-2). Meanwile, the bone matrix proteins (collagen-I and osteocalcin) were increased gradually (d). During the differentiation process, the osteogenic regulatory proteins (Runx2 and Foxo3a) were upregulated, whereas the adipogenic regulatory protein PPAR $\gamma$ was downregulated. Cambinol could inhibit expressions of the osteogenic regulatory proteins, and resveratrol could promote expressions of these proteins. (e) The expression of Sirtuin1 was changed gently; however, the activity of Sirtuin1/2 showed changing obviously by detection of the actylated tubulin- $\alpha$. Scale bar, $50 \mu \mathrm{m}$

alternative cellular models for investigating the stemness and plasticity of cancer cells in solid tumors. ${ }^{20}$ The $\mathrm{NCl}-\mathrm{H} 446$ cell line is a variant SCLC cell line derived from a patient with SCLC, with amplification of oncogenes, such as c-Myc. ${ }^{21,22}$ Although it has been repeatedly passaged and used as an in vitro model of SCLC, its phenotype and tumorigenicity remain quite stable, suggesting that it is a feasible model for exploring the biological characteristics of SCLC in vitro.

In the current study, we found that the $\mathrm{NCl}-\mathrm{H} 446$ cells persisted in expressing the markers of both neuroectoderm and mesoderm, which is reminiscent of the ecto-MSCs. The ecto-MSCs are thought to derive from neural crest stem cells and could differentiate into bones, cartilage, connective tissue, and neural cells. ${ }^{23-25}$ The neural crest cells could delaminate from the neural epithelium and migrate to their differentiation sites. This process conferring neural crest cells the ability to migrate is known as EMT ${ }^{26,27}$ After EMT and migrating to different organs including lungs, the neural crest cells differentiate into neural cells in peripheral nervous system and several ecto-MSCs. In embryonic development, 

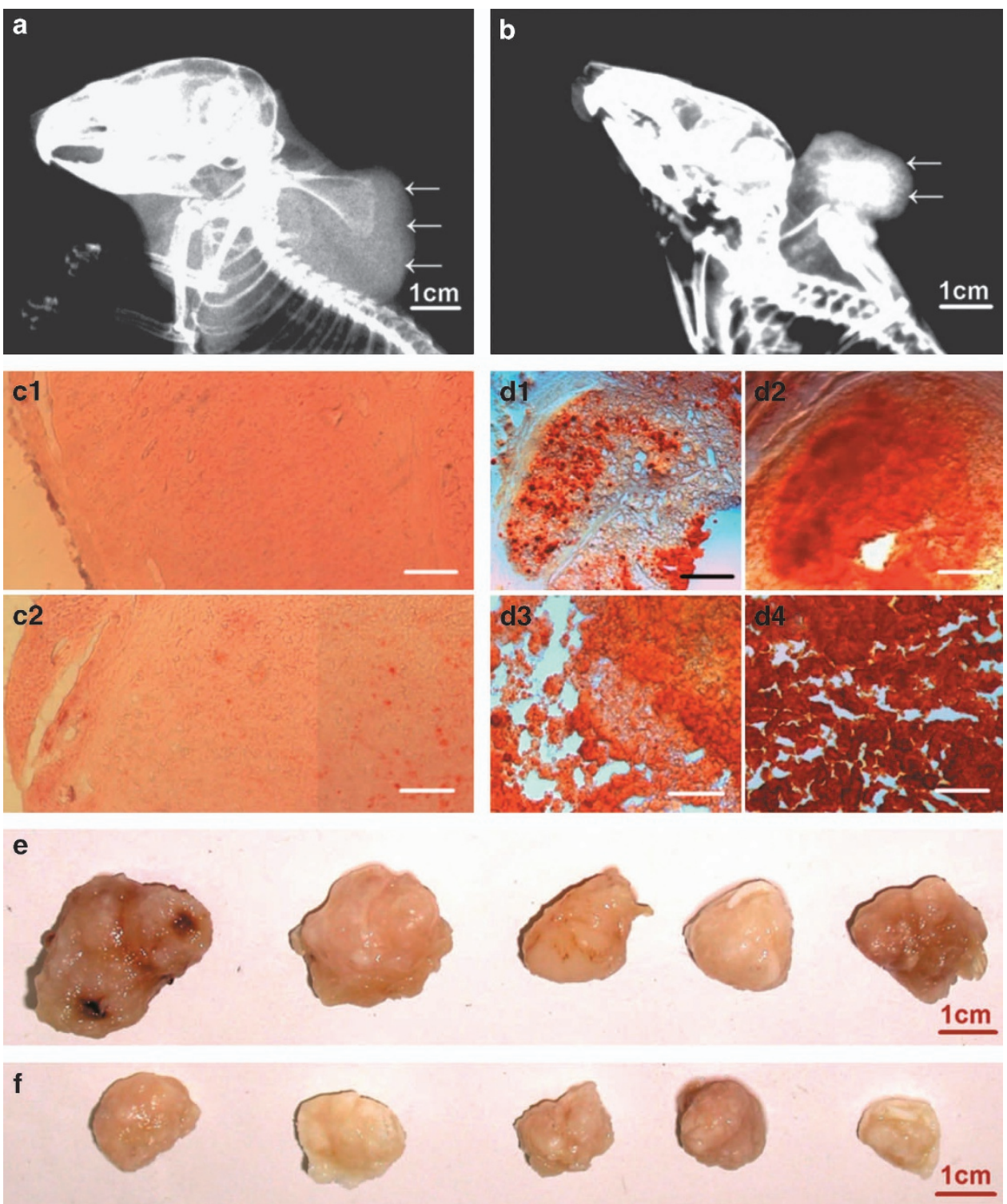

Figure 8 Xenograft tumors were induced to calcify and ceased growth by osteogenic differentiation therapy. (a) In the control group, after injection of complete DMEM medium for 4 weeks, the molybdenum target X-ray radiograph showed that xenograft tumor (arrows) in the back was expanded to soft tissue around the neck of the mouse. The density of the tumor mass was higher than the density of surrounding soft tissue (not showing), and lower than the density of bones. (b) In the osteogenic differentiation group, after injection of osteogenic induction medium for 4 weeks, the tumor mass in the mouse back (arrows) was smaller than that in the control group. The density of the mass was not uniform, and high-density component (calcification) was detected in the center of the mass. (c) The microscopic images of tissue sections, stained with Alizarin Red S, showed that there were fewer small calcified nodules in the xenograft tumors of control animals (c1, injection of complete DMEM medium for 2 week; $\mathbf{c 2}$, injection for 4 weeks). (d) The microscopic images showed that calcium deposition and mineralization in the tumors of treated animals increased gradually (d1-d4, injection of osteogenic induction medium for 1, 2, 3, and 4 weeks, respectively). (e) The xenograft tumors of control animals were over $2 \mathrm{~cm}$ in diameter, and grew for 4 weeks. (f) The xenograft tumors of treated animals were smaller than those in control groups. Scale bar, $1 \mathrm{~cm}(\mathbf{a}, \mathbf{b}, \mathbf{e}$, and $\mathbf{f}) ; 100 \mathrm{~mm}(\mathbf{c}$ and $\mathbf{d})$

neural crest stem cells migrate along mesentery to primitive gut and differentiate to enteric nervous system and pulmonary intrinsic neurons. ${ }^{28,29}$ Although most neural crest cells have differentiated into various kinds of mature cells, there are a few residual cells that still remain undifferentiated in the adult tissues. ${ }^{30-32}$ Undifferentiated cells possessed stemness and could be transformed to malignant cells by oncogenic mutations. Based on the expression of stem cell markers and the cellular phenotype of $\mathrm{NCl}-\mathrm{H} 446$ cells, it is possible that the cancer cells are derived from the pulmonary undifferentiated neural crest cells by malignant transformation. In addition to expressing multilineage stem cell markers, the $\mathrm{NCl}-\mathrm{H} 446$ cells possessed high clonogenicity in anchorage-dependent or -independent conditions in vitro and stable tumorigenicity in vivo, suggesting that most cells of the $\mathrm{NCl}$ $\mathrm{H} 446$ cell line were tumor-initiating cells. These cancer cells in the clones and xenograft tumors expressed various transcription factors and surface molecules of stem cells, which had important roles in initial step of malignant transformation, maintaining the tumor behaviors such as invasiveness, 
metastasis, and acquisition of chemotherapy resistance and EMT phenotype. ${ }^{33-40}$ It has been reported that in the process of EMT, a series of transcription factors, including Snail and c-Myc, which are also the markers of neural crest cells, are required to regulate this process. ${ }^{41-43}$ The cell-surface protein CD44 and MMP-9 could drive EMT and the migration of neural crest cells. ${ }^{4,45}$ The expression of these marker proteins in $\mathrm{NCl}-\mathrm{H} 446$ cells in vitro and in vivo suggests that these cells possess stemness and EMT phenotype, which may confer the SCLC cells with plasticity and malignancy, and promote the cells to disseminate and metastasize to distant organs.

To analyze the plasticity of $\mathrm{NCl}-\mathrm{H} 446$ cells and evaluate the anticancer efficacy of differentiation therapy, these cells were induced to differentiate into ectodermal and mesodermal lineages with various inducing agents in this study. TSA is a hydroxamate-type inhibitor of mammalian HDACs, which can promote the acetylation of histones, and then activate a variety of genes that regulate cell survival, proliferation, differentiation, and apoptosis. ${ }^{46}$ It has been reported that TSA could upregulate the expression of BM88/CEND1 (cell cycle exit and neural differentiation protein 1) in neuroblastoma, which was derived from neural crest stem cells, and induce the cancer cells to exit cell cycle, differentiate, and then undergo apoptosis. ${ }^{47,48}$ Our previous investigation has indicated that TSA can induce $\mathrm{NCl}-\mathrm{H} 446$ cells to undergo neurogenic differentiation and apoptosis. ${ }^{49}$ These results suggested that the SCLC might derive from neural crest cells. Based on the origin of the cancer cells, we thought that the cancer cells should additionally differentiate into mesenchymal cell types, such as adipocyte and osteocyte. For detecting the ability of multidifferentiation, the $\mathrm{NCl}-\mathrm{H} 446$ cells were cultured and induced in adipogenic and osteogenic induction medium, respectively. After adipogenic induction, these cells upregulated expressions of adipogenic regulatory proteins including C/EBP $\beta$ and PPAR $\gamma,{ }^{50}$ and the markers of adipocyte including FAS and adiponectin. These adipogenic differentiating cells produced a lot of lipid droplets increasingly, leading to collapse of the cancer cells finally. It is suggested that unlimited accumulation of lipid droplets in the cancer cells would occupy most space in the cytoplasm and disturb the functions of other organelles, which could lead to lipoptosis of the differentiated cancer cells due to lipotoxicity. ${ }^{51,52}$ Likewise, osteogenic induction could drive the cancer cells to transform into osteoblast-like cells. These differentiated cells showed strong activity of alkaline phosphatase and overexpressions of the osteogenic regulatory proteins, inducing Runx2 and Foxo3a, and bone matrix proteins, which had important roles in osteogenic differentiation and ossification. $^{53,54}$ As calcium deposition, on the surface of the differentiating cancer cells and in the ECM (osteoid), increased gradually, the cancer cells were buried into the cement-like bone matrix, suggesting that inducing calcification will be an efficient strategy for forcing autophagy or/and apoptosis of the cancer cells due to the stress of hypoxia and poor nutrition. To explore the mechanisms of inducing differentiation of the cancer cells, the expression and cleavage of the regulatory proteins for autophagy and apoptosis were analyzed in the processes of inducing neurogenic and osteogenic differentiation. The detections of these proteins showed that in the stress of inducing differentiations, the cancer cells underwent both autophagy and apoptosis. It has been reported that there was crosstalking between autophagy and apoptosis, ${ }^{55,56}$ in murine hematopoietic cell line, the activated caspase-3, by withdrawal of growth factors, could cleave Beclin-1, and then the C-terminal fragment of Beclin-1 enhanced apoptosis. ${ }^{57,58}$ In this study, the results of western blotting showed that the Atg7, Beclin-1, and capased-3 were cleaved, and LC3-I was processed into LC3-II as well, suggesting the cross-talking between autophagy and apoptosis during the processes of neurogenic and osteogenic differentiations in NCl-H446 cells. In addition, to explore the functions of $\mathrm{NAD}^{+}$-dependent deacetylases, Sirtuins, which have been reported to regulate the osteogenic/adipogenic differentiation of normal stem cells, ${ }^{59,60}$ the effects of the inhibitor or agonist for Sirt $1 / 2$ on the process in the $\mathrm{H} 446$ cells was investigated. The results indicated that activation of Sirt1/2 could promote the differentiation of these cancer cells, suggesting Sirt1/2 as the novel target for treating cancer cells by inducing osteogenic/adipogenic differentiation.

Based on these results in vitro, to evaluate the potential application of the inducing differentiation for translational clinical therapy to treat cancers, the preliminary trails in vivo of osteogenic differentiation therapy for treating the mice bearing xenograft tumors were arranged finally. The results demonstrated that after this treatment, subcutaneous xenograft tumors could be induced to calcify and ceased growth, suggesting that the differentiation therapy for SCLC is efficient in vivo as well. However, inducing multilineage differentiations of the cancer cells in SCLC xenograft tumors and understanding the mechanisms, in vivo, responsible for the efficiency of this therapy remain to be elucidated in the future work.

\section{Conclusions}

Most cancer cells in the SCLC NCl-H446 cell line shared the phenotypic characteristics of both neuroectoderm and mesoderm lineages. These cells possessed high stemness, tumorigenicity, and plasticity, and could be induced to terminally differentiate and undergo autophagy and apoptosis by various inducing agents. These findings have potentially some translational applications in treatments of SCLC with inducing differentiation therapy. This therapy will possess some advantages compared with the routine chemical therapy, such as lower systemic toxicity and high efficiently targeting cancer stem cells.

\section{Materials and Methods}

Cancer cell line and reagents. SCLC NCI-H446 cell line (supplied by American Type Culture Collection, Manassas, VA, USA) was purchased from the cell bank of Shanghai Institute of Biochemistry and Cell Biology (Shanghai, China). DMEM medium, Neurobasal medium, and FBS were purchased from Invitrogen (Paisley, UK). Goat polyclonal anti-Oct4; rabbit polyclonal anti-Sall-4, anti-Sox-2, anti-c-Myc, anti-Bax, anti-PPAR $\gamma$, anti-C/EBP $\beta$, anti-FAS, anti-adiponectin, anticollage-I, anti-Runx2, anti-Foxo3a, anti-Sirtuin-1, and anti-active + pro-caspase-3; mouse monoclonal anti-Bcl-2, anti-CD44, anti-Vimentin, anti-Ki67, anti-Snail + Slug, anti-endoglin (CD105), anti-NF-200, and anti-Osteocalcin; and rabit monoclonal anti-Atg7, anti-Beclin-1, and anti-LC3B antibodies were purchased from Abcam (Cambridge, UK). Goat polyclonal anti-CD133; rabbit polyclonal antiNCAM, anti-BM88; and mouse monoclonal anti-MMP-9 antibodies were 
purchased from Santa Cruz Biotechnology (Santa Cruz, CA, USA). Rabbit polyclonal anti-Nestin and mouse monoclonal anti-S100 $\beta$, anti-acetyl-tubulin- $\alpha$, anti- $\beta$-tubulin, and anti- $\beta$-actin antibodies were purchased from Sigma-Aldrich (St. Louis, MO, USA). Rabbit polyclonal anti-acetyl-H3K9 and mouse monoclonal antihistone $\mathrm{H} 3$ antibodies were purchased from Cell Signaling (Danvers, MA, USA). Cy3-conjugated second antibodies, ATRA, TSA, dexamethasone, $\beta$-glycerophosphate disodium, L-ascorbic acid 2-phosphate, insulin, indomethacin, 3-isobutyl-1methyl-xanthine (IBMX), resveratrol, cambinol, Alizarin Red S, and Oil Red O were purchased from Sigma-Aldrich. BALB/C-nude mice were purchased from the Shanghai Experimental Animal Center (Chinese Academy of Sciences, Shanghai, China).

Cell culture and detection of stem cell markers. $\mathrm{NCl}-\mathrm{H} 446$ cells were cultured in DMEM medium containing $10 \% \mathrm{FBS}$ at $37^{\circ} \mathrm{C}$ with $5 \% \mathrm{CO}_{2}$ and $100 \%$ humidity. For detecting expression of stem cell markers, the passaged cancer cells were cultured on plastic plates or laminin-coated glass coverslips, for 2 days, fixed using $4 \%$ paraformaldehyde, and then immunofluorescence-stained with antibodies against cancer stem cell marker CD133, pluripotent stem cell markers Sall4 and Oct4, neural crest stem cell markers Nestin, NCAM, and S100 $\beta$, and MSC markers Vimentin, CD44, and CD105. These stained cancer cells were observed with immunofluorescence microscope (Axio Observer; ZEISS, Yena, Germany).

Clonogenicity analyses. For adherent colony formation assay, $\mathrm{NCl}-\mathrm{H} 446$ cells passaged in flasks were harvested by $0.25 \%$ trypsin in logarithmic growth phase and centrifuged at 1000 r.p.m. for $5 \mathrm{~min}$. The cells were resuspended in fresh medium, diluted to $10 \mathrm{cell} / \mathrm{s} / \mathrm{ml}$ using limiting dilution procedures, seeded into 96-well plates $(0.1 \mathrm{ml} /$ well, contained about one cell), and cultured in DMEM medium containing $10 \% \mathrm{FBS}$. Wells containing no cell or more than one cell were excluded, and the wells with only one cell were marked and observed daily under phase contrast microscope (Axio Observer; ZEISS). After culture for 1 week, the clones were counted, dissociated, and cultured as above in new 96-well culture plates ( 1 cell/well) to generate subclones. The primary clones and subclones at different growth stages were fixed using $4 \%$ paraformaldehyde, and then immunofluorescence-stained with the antibody against neural crest marker NCAM. To analyze the stemness and plasticity, the subclones were cultured in Neurobasal medium containing 2\% B27 (Invitrogen), $10 \mu \mathrm{M}$ ATRA, and $2 \mathrm{mM}$ glutamine for inducing the cells differentiation to neurons. The differentiated cells were immunofluorescence-stained with the antibody against neuron marker NF-200. For colony formation assay in anchorage-independent condition, six-well culture plates were first coated with $0.7 \%$ agarose (dissolved in DMEM contained $10 \% \mathrm{FBS}$ ), and after polymerization of this agarose (base agarose gel), the cancer cells were resuspended at very low density ( $1500 \mathrm{cells} / 1.5 \mathrm{ml}$ ) in $0.35 \%$ agarose (dissolved in DMEM contained 10\% FBS) and added onto the base agarose gel. After polymerization of the upper agarose in about $15 \mathrm{~min}, 2 \mathrm{ml}$ of medium was added. These cells in upper agarose were cultured for 3 weeks, the medium was changed every 5 days. The colonies were dynamically observed with phase contrast microscope (Axio Observer; ZEISS). Some colonies were picked out and implanted on to the polylysine-coated glass coverslips in 24-well culture plates and cultured with DMEM containing 10\% FBS. After culture for different time, these colonies were immunofluorescence-stained with the antibody against neural crest marker NCAM.

Tumorigenicity assay. When the $\mathrm{NCl}-\mathrm{H} 446$ cells cultured with complete DMEM medium in the flasks covered $70 \%$ of the bottom, the cells were digested with $0.25 \%$ trypsin and then passaged for expansion. Finally, the cancer cells were harvested, and then $1 \times 10^{4}$ cells were injected subcutaneously into the left front dorsum of 10 4-week-old female BALB/C-nude mice, which were examined visually every 3 days. When the subcutaneous xenograft tumors grew to $1 \mathrm{~cm}$ in diameter (after injection about for 2 weeks), three mice were killed under deep anesthesia with pentobarbital. The tumors were dissected and subjected to mechanical and enzymatic dissociation. The digested cancer cells were further sieved through a $100-\mu \mathrm{m}$ cell strainer to obtain single-cell suspension. These cancer cells derived from subcutaneous xenograft tumors were cultured in flasks for expansion and second clonogenicity analysis. For testing the stableness of tumorigenicity of cancer cells in the solid tumors, $1 \times 10^{4}$ cells of one subclone derived from subcutaneous xenograft tumor were injected into the left lungs of 10 mice to secondly generate orthotopic xenograft tumors. After injection of cancer cells for 1,3 , and 5 weeks, the mice bearing subcutaneous or orthotopic xenograft tumors were killed under deep anesthesia with pentobarbital. These xenograft tumors with surrounding tissue were dissected and then fixed with $4 \%$ paraformaldehyde. These procedures above received ethical approval from the Jiangsu University Animal Care and Use Committee and were consistent with the animal care guidelines. The xenograft tumors, at different growth stages, with surrounding tissues, were sectioned using a freezing microtome (CM1900; Leica, Solms, Germany), and the sections were immunofluorescence-stained or immunohistochemically stained with the antibody against SCLC marker NCAM or proliferation cell nuclear antigen Ki67 for showing the growth property of the cancer cells in normal tissues and organs of the animals. To analyze stem cell phenotype of the cancer cells in vivo, the sections of subcutaneous xenograft tumors were additionally immunofluorescence-stained with the antibodies against stem cell markers, including CD133, CD44, Nestin, Vimentin, Sox-2, Snail, and c-Myc, metastasis-associated protein MMP-9, and proliferation cell nuclear antigen Ki67. After staining, these histological slides were observed with immunofluorescence microscope (Axio Observer; ZEISS).

Neurogenic differentiation assay. To analyze the cellular plasticity and effects of differentiation therapy on $\mathrm{NCl}-\mathrm{H} 446$ cells, the cancer cells cultured in DMEM medium containing 10\% FBS were treated with $500 \mathrm{nM}$ TSA for 1 week. The medium was replaced every 3 days. After inducing differentiation for different time, the neuron-like cancer cells were fixed using $4 \%$ paraformaldehyde before immunofluorescence staining with antibodies against neuron-specific markers BM88 and NF-200. To evaluate the effects of TSA-induced differentiation on proliferation, autophagy, and apoptosis of $\mathrm{NCl}-\mathrm{H} 446$ cells, the immunofluorescence staining for Ki67 and Beclin-1 and the TUNEL methods were applied to examine proliferating, autophagic, and apoptotic cancer cells, respectively. In addition, the expressions of neuron markers BM88 and NF-200, autophagy-related proteins including Atg7, Beclin-1, and LC3-II/LC3-I, and apoptosis-associated proteins including $\mathrm{Bax}, \mathrm{Bcl}-2$, and caspase-3 were detected with western blotting. The acetyl-H3K9 were also detected for evaluating the effect of TSA on inhibition of HDACs.

Adipogenic differentiation assay. $\mathrm{NCl}-\mathrm{H} 446$ cells were cultured in DMEM medium supplemented with $10 \%$ FBS, $2 \mu \mathrm{M}$ insulin, $500 \mu \mathrm{M}$ IBMX, $1 \mu \mathrm{M}$ dexamethasone, and $200 \mu \mathrm{M}$ indomethacin for 3 days. This medium was referred here as 'induction medium'. Subsequently, the cells were cultured in maintenance medium containing $2 \mu \mathrm{M}$ insulin for 1 day, and then cultured in induction medium again. The inducing cycle was repeated three times. At the end of inducing differentiation, adipocyte-like cancer cells were observed by phase contrast microscope (Axio Observer; ZEISS) and stained with Oil Red $O$ to reveal lipid droplets in these cells. The expressions of adipogenic regulatory proteins including $\mathrm{C} / \mathrm{EBP} \beta$ and PPAR $\gamma$, and the markers of adipocyte including FAS and adiponectin, were detected with western blotting.

Osteogenic differentiation assay. $\mathrm{NCl}-\mathrm{H} 446$ cells were cultured in osteogenic induction medium (DMEM supplemented with 10\% FBS, $0.1 \mu \mathrm{M}$ dexamethasone, $10 \mathrm{mM} \beta$-glycerophosphate disodium, and $0.2 \mathrm{mM}$ L-ascorbic acid 2-phosphate) for 3 weeks. The medium was supplemented with $100 \mu \mathrm{M}$ cambinol or $100 \mu \mathrm{M}$ resveratrol, for analyzing the effects of Sirt1/2 on osteogenic differentiation. The osteogenic activity of induced cancer cells was tested by alkaline phosphatase activity with NBT staining. The efficiency of ossification of differentiated cancer cells was analyzed by detection of the bone nodules on the surface of induced osteoblasts with Alizarin Red $S$ staining and observation by phase contrast microscope (Axio Observer; ZEISS). To explore the mechanisms of osteogenic differentiation, the expressions of osteogenic/adipogenic regulatory proteins, inducing Runx2, Foxo3a, and PPAR $\gamma$, bone matrix proteins including collagen-I and osteocalcin, and autophagy/apoptosis-associated proteins including Beclin-1, LC3-II/LC3-I, Bax, Bcl-2, and caspase-3 were detected with western blotting. The levels of Sirt1 and actylated tubulin- $\alpha$ were also detected for evaluating the effect of cambinol or resveratrol on the expression and activity of Sirt1/2.

Experiments of osteogenic differentiation therapy in vivo. The procedure of implanting subcutaneous xenograft tumor in animals was the same as above. When the subcutaneous xenograft tumors grew to $1 \mathrm{~cm}$ in diameter, these mice (nine mice in the control group, nine mice in treatment group) bearing subcutaneous tumor were anesthetized with sodium pentobarbital. The complete DMEM medium (as control) or osteogenic induction medium (as differentiation 
therapy) was injected into subcutaneous xenograft tumors and surrounding tissues, respectively, for 4 weeks ( $100 \mu$ l every 2 days, for 2 weeks; followed $200 \mu \mathrm{l}$ every 2 days, for 2 weeks). During the period, some mice were examined with molybdenum target X-ray every 2 weeks, using the Mammography Unit (GIOTTO IMAGE Company, Bologna, Italy), which provided with the specificity and sensitivity for detecting the calcification component in tissue and organs. The operation parameters of X-ray examination were as follows: Tube voltage: $25 \mathrm{KV}$; maximum absorbed current: $20 \mathrm{~A}$; exposure time: $65 \mathrm{~mA} / \mathrm{s}$; and operating power: $3.5 \mathrm{KW}$. After treatments for different time, these mice were killed under deep anesthesia with pentobarbital. The subcutaneous xenograft tumors were dissected and fixed with $4 \%$ paraformaldehyde. The frozen sections of the xenograft tumors were prepared and stained by Alizarin Red S for visualizing calcium deposition and were observed with light microscope (Axio Observer; ZEISS).

Immunoblotting. NCl-H446 cells treated with or without inducing differentiation were lysed using RIPA Lysis Buffer containing protease inhibitor cocktail (Santa Cruz Biotechnology). The concentration of proteins was determined by a BCA kit (Thermo Fisher Scientific, Rockford, IL, USA). Equal protein amount from each sample was loaded onto $8 \%$ polyacrylamide gels, separated by SDS-PAGE, and transferred to a polyvinylidene difluoride (PVDF) membrane (Millipore, Temecula, CA, USA) by electrophoresis. The PVDF membrane was incubated with the antibodies against special proteins and $\beta$-actin (standard control) followed by HRP-conjugated second antibodies (Santa Cruz Biotechnology). The protein bands were visualized using Pierce ECL Plus Substrate (Thermo Fisher Scientific) and scanned with Typhoon 9400 Variable Mode Imager (Amersham Biosciences, Little Chalfont, UK).

\section{Conflict of Interest}

The authors declare no conflict of interest.

Acknowledgements. This work was supported by the Major Research Plan of the National Natural Science Foundation of China (Grant No. 91129718), the National Natural Science Foundation of China (Grant No. 81071421 and 81170573), Jiangsu Province's Project of Scientific and Technological Innovation and Achievements Transformation (Grant No. BL2012055), Jiangsu Province's Outstanding Medical Academic Leader and Sci-tech Innovation Team Program (Grant No.LJ201117), and the Natural Science Foundation of the Jiangsu Province (Grant No.BK2012709).

1. Rodriguez E, Lilenbaum RC. Small cell lung cancer: past, present, and future. Curr Oncol Rep 2010; 12: 327-334.

2. Demedts IK, Vermaelen KY, van Meerbeeck JP. Treatment of extensive-stage small cell lung carcinoma: current status and future prospects. Eur Respir J 2010; 35: 202-215.

3. Kalemkerian GP. Advances in the treatment of small-cell lung cancer. Semin Respir Crit Care Med 2011; 32: 94-101.

4. Gridelli C, Rossi A, Airoma G, Bianco R, Costanzo R, Daniele B et al. Treatment of pulmonary neuroendocrine tumours: State of the art and future developments. Cancer Treat Rev 2013; 39: 466-472.

5. Sell S. Cancer stem cells and differentiation therapy. Tumour Biol 2006; 27: 59-70.

6. Sell S. On the stem cell origin of cancer. Am J Pathol 2010; 176: 2584-2594.

7. Sell S, Pierce GB. Maturation arrest of stem cell differentiation is a common pathway for the cellular origin of teratocarcinomas and epithelial cancers. Lab Invest 1994; 70: 6-22.

8. Tontonoz P, Singer S, Forman BM, Sarraf P, Fletcher JA, Fletcher CD et al. Terminal differentiation of human liposarcoma cells induced by ligands for peroxisome proliferatoractivated receptor gamma and the retinoid X receptor. Proc Natl Acad Sci USA 1997; 94: 237-241.

9. Samid D, Hudgins WR, Shack S, Liu L, Prasanna P, Myers CE. Phenylacetate and phenylbutyrate as novel, nontoxic differentiation inducers. Adv Exp Med Biol 1997; 400A: 501-505.

10. Wang ZY, Chen Z. Acute promyelocytic leukemia: from highly fatal to highly curable. Blood 2008; 111: 2505-2515.

11. Fenaux $P$, Chevret $S$, Guerci A, Fegueux N, Dombret H, Thomas $X$ et al. Long-term followup confirms the benefit of all-trans retinoic acid in acute promyelocytic leukemia. European APL group. Leukemia 2000; 14: 1371-1377.

12. Campos B, Wan F, Farhadi M, Ernst A, Zeppernick F, Tagscherer KE et al. Differentiation therapy exerts antitumor effects on stem-like glioma cells. Clin Cancer Res 2010; 16: 2715-2728.

13. Seigel GM. Differentiation potential of human retinoblastoma cells. Curr Pharm Biotechnol 2011; 12: 213-216.
14. Kawamata $H$, Tachibana M, Fujimori $T$, Imai $Y$. Differentiation-inducing therapy for solid tumors. Curr Pharm Des 2006; 12: 379-385.

15. Sell S. Stem cell origin of cancer and differentiation therapy. Crit Rev Oncol Hematol 2004; 51: 1-28.

16. Park KS, Liang MC, Raiser DM, Zamponi R, Roach RR, Curtis SJ et al. Characterization of the cell of origin for small cell lung cancer. Cell Cycle 2011; 10: 2806-2815.

17. Sutherland KD, Proost N, Brouns I, Adriaensen D, Song JY, Berns A. Cell of origin of small cell lung cancer: inactivation of Trp53 and Rb1 in distinct cell types of adult mouse lung. Cancer Cell 2011; 19: 754-764.

18. Calbo J, van Montfort E, Proost N, van Drunen E, Beverloo HB, Meuwissen R et al. A functional role for tumor cell heterogeneity in a mouse model of small cell lung cancer. Cancer Cell 2011; 19: 244-256.

19. Liu Y, Zhang N, Wang Y, Xu M, Liu N, Pang X et al. Zinc finger E-box binding homeobox 1 promotes invasion and bone metastasis of small cell lung cancer in vitro and in vivo. Cancer Sci 2012; 103: 1420-1428.

20. Kondo T. Stem cell-like cancer cells in cancer cell lines. Cancer Biomarkers 2007; 3 : 245-250.

21. Carney DN, Gazdar AF, Bepler G, Guccion JG, Marangos PJ, Moody TW et al. Establishment and identification of small cell lung cancer cell lines having classic and variant features. Cancer Res 1985; 45: 2913-2923.

22. Gazdar AF, Carney DN, Nau MM, Minna JD. Characterization of variant subclasses of cell lines derived from small cell lung cancer having distinctive biochemical, morphological, and growth properties. Cancer Res 1985; 45: 2924-2930.

23. Blentic A, Tandon P, Payton S, Walshe J, Carney T, Kelsh RN et al. The emergence of ectomesenchyme. Dev Dyn 2008; 237: 592-601.

24. Yan Z, Lin Y, Jiao X, Li Z, Wu L, Jing W et al. Characterization of ectomesenchymal cells isolated from the first branchial arch during multilineage differentiation. Cells Tissues Organs 2006; 183: 123-132.

25. Widera D, Zander C, Heidbreder M, Kasperek Y, Noll T, Seitz $\mathrm{O}$ et al. Adult palatum as a novel source of neural crest-related stem cells. Stem Cells 2009; 27: 1899-1910.

26. Strobl-Mazzulla PH, Bronner ME. Epithelial to mesenchymal transition: New and old insights from the classical neural crest model. Semin Cancer Biol 2012; 22: 411-416.

27. Bronner ME. Formation and migration of neural crest cells in the vertebrate embryo. Histochem Cell Biol 2012; 138: 179-186.

28. Langsdorf A, Radzikinas K, Kroten A, Jain S, Ai X. Neural crest cell origin and signals for intrinsic neurogenesis in the mammalian respiratory tract. Am J Respir Cell Mol Biol 2011; 44: 293-301.

29. Burns AJ, Delalande JM. Neural crest cell origin for intrinsic ganglia of the developing chicken lung. Dev Biol 2005; 277: 63-79.

30. Burns AJ, Pasricha PJ, Young HM. Enteric neural crest-derived cells and neural stem cells: biology and therapeutic potential. Neurogastroenterol Motil 2004; 1(Suppl): 3-7.

31. Kruger GM, Mosher JT, Bixby S, Joseph N, Iwashita T, Morrison SJ. Neural crest stem cells persist in the adult gut but undergo changes in self-renewal, neuronal subtype potential, and factor responsiveness. Neuron 2002; 35: 657-669.

32. Trentin A, Glavieux-Pardanaud C, Le Douarin NM, Dupin E. Self-renewal capacity is a widespread property of various types of neural crest precursor cells. Proc Natl Acad Sci USA 2004; 101: 4495-4500.

33. Chen S, Xu Y, Chen Y, Li X, Mou W, Wang L et al. SOX2 gene regulates the transcriptional network of oncogenes and affects tumorigenesis of human lung cancer cells. PLOS One 2012; 7: $\mathrm{e} 36326$.

34. Shih JY, Yang PC. The EMT regulator slug and lung carcinogenesis. Carcinogenesis 2011; 32: 1299-1304.

35. Kobayashi D, Kuribayshi K, Tanaka M, Watanabe N. SALL4 is essential for cancer cell proliferation and is overexpressed at early clinical stages in breast cancer. Int J Oncol 2011; 38: 933-939.

36. Li X, Wang J, Xu Z, Ahmad A, Li E, Wang Y et al. Expression of sox2 and oct4 and their clinical significance in human non-small-cell lung cancer. Int J Mol Sci 2012; 13: 7663-7675.

37. Kobayashi D, Kuribayashi K, Tanaka M, Watanabe N. Overexpression of SALL4 in lung cancer and its importance in cell proliferation. Oncol Rep 2011; 26: 965-970.

38. Light W, Vernon AE, Lasorella A, lavarone A, LaBonne C. Xenopus Id3 is required downstream of Myc for the formation of multipotent neural crest progenitor cells. Development 2005; 132: 1831-1841.

39. Shi C, Tian R, Wang M, Wang X, Jiang J, Zhang Z et al. CD44 + CD133 + population exhibits cancer stem cell-like characteristics in human gallbladder carcinoma. Cancer Biol Ther 2010; 10: 1182-1190.

40. Bellizzi A, Sebastian S, Ceglia P, Centonze M, Divella R, Manzillo EF et al. Co-expression of CD133( + )/CD44( + ) in human colon cancer and liver metastasis. J Cell Physiol 2013; 228: 408-415.

41. del Barrio MG, Nieto MA. Overexpression of Snail family members highlights their ability to promote chick neural crest formation. Development 2002; 129: 1583-1593.

42. Hong SK, Tsang M, Dawid IB. The mych gene is required for neural crest survival during zebrafish development. PLoS One 2008; 3: e2029.

43. Bellmeyer A, Krase $\mathrm{J}$, Lindgren $\mathrm{J}$, LaBonne $\mathrm{C}$. The protooncogene c-myc is an essential regulator of neural crest formation in xenopus. Dev Cell 2003; 4: 827-839.

44. Ikeda K, Nakao J, Asou H, Toya S, Shinoda J, Uyemura K. Expression of CD44H in the cells of neural crest origin in peripheral nervous system. Neuroreport 1996; 7: 1713-1716. 
45. Monsonego-Ornan E, Kosonovsky J, Bar A, Roth L, Fraggi-Rankis V, Simsa S et al. Matrix metalloproteinase 9/gelatinase B is required for neural crest cell migration. Dev Biol 2012; 364: 162-177.

46. Meng J, Zhang HH, Zhou CX, Li C, Zhang F, Mei QB. The histone deacetylase inhibitor trichostatin $A$ induces cell cycle arrest and apoptosis in colorectal cancer cells via p53dependent and -independent pathways. Oncol Rep 2012; 8: 84-88.

47. Jiang M, Stanke J, Lahti JM. The connections between neural crest development and neuroblastoma. Curr Top Dev Biol 2011; 94: 77-127.

48. Politis PK, Akrivou S, Hurel C, Papadodima O, Matsas R. BM88/Cend1 is involved in histone deacetylase inhibition-mediated growth arrest and differentiation of neuroblastoma cells. FEBS Lett 2008; 582: 741-748.

49. Liu K, Ge ZJ, Chen L, Chen RH, Zhang ZJ, Zhou YP et al. Effects of trichostanin in inducing differentiation and apoptosis of small cell lung cancer $\mathrm{NCl}-\mathrm{H} 446$ cell line. Jiangsu Med $\mathrm{J}$ 2012; 38: 1010-1013.

50. Ni Y, Ji C, Wang B, Qiu J, Wang J, Guo X. A novel pro-adipogenesis factor abundant in adipose tissues and over-expressed in obesity acts upstream of PPAR $\gamma$ and $\mathrm{C} / \mathrm{EBP} \alpha$ J Bioenerg Biomembr 2012; e-pub ahead of print 13 December 2012.

51. Jean E. Schaffer. Lipotoxicity: when tissues overeat. Curr Opin Lipido 2003; 14: 281-287.

52. Pohle T, Brändlein S, Ruoff N, Müller-Hermelink HK, Vollmers HP. Lipoptosis: tumorspecific cell death by antibody-induced intracellular lipid accumulation. Cancer Res 2004; 64: 3900-3906.

53. Liu TM, Lee EH. Transcriptional regulatory cascades in runx2-dependent bone development. Tissue Eng Part B Rev 2012; 19: 254-263.

54. Tseng PC, Hou SM, Chen RJ, Peng HW, Hsieh CF, Kuo ML et al. Resveratrol promotes osteogenesis of human mesenchymal stem cells by upregulating RUNX2 gene expression via the SIRT1/FOXO3A axis. J Bone Miner Res 2011; 26: 2552-2563.
55. Kapuy O, Vinod PK, Mandl J, Bánhegyi G. A cellular stress-directed bistable switch controls the crosstalk between autophagy and apoptosis. Mol Biosyst 2013; 9: 296-306.

56. Gordy $\mathrm{C}, \mathrm{He}$ YW. The crosstalk between autophagy and apoptosis: where does this lead? Protein Cell 2012; 3: 17-27.

57. Kang R, Zeh HJ, Lotze MT, Tang D. The Beclin 1 network regulates autophagy and apoptosis. Cell Death Differ 2011; 18: 571-580.

58. Wirawan E, Vande Walle L, Kersse K, Cornelis S, Claerhout S, Vanoverberghe I et al. Caspase-mediated cleavage of Beclin-1 inactivates Beclin-1-induced autophagy and enhances apoptosis by promoting the release of proapoptotic factors from mitochondria. Cell Death Dis 2010; 1: e18.

59. Srivastava S, Bedi U, Roy P. Synergistic actions of insulin-sensitive and Sirt1-mediated pathways in the differentiation of mouse embryonic stem cells to osteoblast. Mol Cell Endocrinol 2012; 361: 153-164.

60. Shakibaei M, Shayan P, Busch F, Aldinger C, Buhrmann C, Lueders C et al. Resveratrol mediated modulation of Sirt-1/Runx2 promotes osteogenic differentiation of mesenchymal stem cells: potential role of Runx2 deacetylation. PLoS One 2012; 7: e35712.

(2) (3)(2) Cell Death and Disease is an open-access journal BY $\mathrm{NC}$ sA published by Nature Publishing Group. This work is licensed under a Creative Commons Attribution-NonCommercialShareAlike 3.0 Unported License. To view a copy of this license, visit http://creativecommons.org/licenses/by-nc-sa/3.0/ 